\title{
Research on Clearance of Aerial Remote Sensing Images Based on Image Fusion
}

\author{
Yingying Gai ${ }^{1}$ \\ Institute of Oceanographic Instrumentation, Shandong Academy of Sciences \\ Qingdao, 266061, China \\ E-mail:gyygyy12340163.com

\section{Zhigang Gai $^{2}$} \\ Institute of Oceanographic Instrumentation, Shandong Academy of Sciences \\ Qingdao, 266061, China \\ E-mail:441559120@qq.com
}

\section{Yuan Liu}

Specialized Forces College of CAPF

Beijing, 102202, china

E-mail:2008.1iuyuan.2008@163.com

\section{Enxiao Liu}

Institute of Oceanographic Instrumentation, Shandong Academy of Sciences

Qingdao, 266061, China

E-mail:93228047@qq.com

Dark channel prior defogging algorithm can not only effectively remove the influence of clouds on the aerial remote sensing images, but also bring incomplete defogging to compensate the lack of image details recovery or even covering and so on. In order to slove this problem, an image fusion scheme is proposed in this paper. Based on the combination of the dark channel prior and guided filtering, the LOG edge detection features of the original images and defogging results will be fused at a certain proportion. Through the experimental analysis and comparison of a large number of remote sensing images, we find that the amount of image information becomes larger after fusion and the percentage of entropy reaches a maximum value and then decreases as the fusion ratio increases. In view of this, the optimal fusion ratio of the images is determined. Based on the subjective and objective evaluation results of the image quality, the correctness and validity of the method are verified. Therefore, this method not only removes the clouds effectively, but also retains the details of the original images to achieve the purpose of image clearness.

CENet2017

22-23 July 2017

Shanghai, China

\footnotetext{
${ }^{1}$ Speaker and Corresponding Author

${ }^{2}$ This study is supported by Special Fund for Marine Public Welfare Scientific Research (201505031) and Qingdao Entrepreneurship and Innovation Leading Talent Project (13CX-24)
} 


\section{Introduction}

As an indispensable means to detect and study the earth resources and environment, aerial remote sensing has the advantages of large imaging scale, high spatial resolution and real-time imaging. It is widely used in large area terrain mapping and small area investigation and so on. However, since it is often affected by clouds and fog, the dynamic range of gray level of the image is narrowed, and also the clarity of image is reduced and so are the accuracy of identification and extraction of features [1]. Therefore, the application of aerial remote sensing is very important to remove the influence of clouds, and strengthen the detailed features of the objects so as to improve the image clarity and utilization.

In recent years, many researchers have studied the image degradation mechanism under bad weather conditions. For example, Galdran et al. proposed a fusion scheme to combine the output of the minimization of two energy functionals of foggy images, and achieved good effects on both nearby regions and faraway regions [2]. Lee et al. combined a defogging algorithm with a demosaicking algorithm and achieved better defogging performance with fewer artifacts [3]. After statistical analysis of a large number of outdoor fog-free images, Kaiming $\mathrm{He}$ et al. proposed a dark channel prior method to remove fog and obtained good results [4]. Also, as the most practical and effective method,this method used a soft matching algorithm to optimize the transmittance, so, the execution speed is slow and the fog removal efficiency is low. In order to obtain the real-time defogging effect, Kaiming He et al. used a guided filtering method instead of the soft matching method [5]. However, it is easy to bring incomplete defogging, and the details of the image features still cannot be highlighted to some extent [6]. In addition, the colors of some images are changed after the fog removal, and some texture features are covered due to local contrast changes. Remote sensing image application requires high standard of image texture and edge information, so it is necessary to strengthen the texture and edge of the image to improve the image clarity.

In view of the above problems, a new method based on dark channel prior and the Laplacian of Gaussian (LOG) edge detection is proposed in this paper. First, the edges of the original images are obtained, and then the edge detection results are fused with the dark channel prior defogged results in a certain proportion. In this way, the clouds and fog are removed effectively, and the processed images have the same characteristics on the edges as the foggy images achieve the purpose of rapid image clearance.

\section{Dark channel prior defogging}

\subsection{Imaging model of foggy images}

According to the Mc Cartney model [7], the attenuation term and the atmospheric light imaging term exist simultaniously and play a leading role in the atmospheric scattering model in foggy weather, which leads to the reduction of contrast and resolution of the image [8]. The imaging model of the foggy image can be described as:

$$
I(x)=J(x) t(x)+A(1-t(x))
$$

Where $I(x)$ is the observed intensity, $J(x)$ is the scene radiance, $A$ is the global atmospheric light, $t(x)$ is the medium transmission describing the portion of the light that is 
not scattered and reaches the camera. The first term in the equation is called direct attenuation, and the second term is called airlight.

\subsection{Preliminary estimate of transmittance}

For an arbitrary image $J$, its dark channel is given by

$$
J^{\text {dark }}(x)=\min _{c \in\{r, g, b\}}\left(\min _{y \in \Omega(x)} J^{c}(y)\right) \rightarrow 0
$$

where $J^{c}$ is a color channel of $J$ and $\Omega(x)$ is a local patch centered at $x$. we can estimate the transmission $\tilde{t}(x)$ simply by

$$
\tilde{t}(x)=1-\min _{c \in\{r, g, b\}}\left(\min _{y \in \Omega(x)}\left(\frac{I^{c}(y)}{A^{c}}\right)\right)
$$

we can optionally keep a very small amount of haze for the distant objects by introducing a constant parameter $\omega(0<\omega \leq 1)$ :

$$
\tilde{t}(x)=1-\omega \min _{c \in\{r, g, b\}}\left(\min _{y \in \Omega(x)}\left(\frac{I^{c}(y)}{A^{c}}\right)\right)
$$

where $\omega=0.95$ 。

\subsection{Transmittance optimization}

In order to improve the accuracy, Kaiming He used the guided filtering to estimate the transmittance. Namely, , the input image is filtered by a guided image, so the output image can not only retain the overall characteristics of the input image but also obtain the change details of the guided image [9].

Suppose the foggy image is the guided image $I^{\text {guide }}$, if the guided image is a color image, the transmittance $\tilde{t}(x)$ can be optimized by a guided filter. Suppose the optimized transmittance is $q(x)$, in accordance with the guided filtering principle, $q(x)$ can be considered as the linear transform of all pixels which take $k$ as the center and $w_{k}$ as the window size. It can be expressed as:

$$
q_{i}(x)=a_{k}^{T} I_{i}^{\text {guide }}+b_{k}, \forall i \in w_{k}
$$

where $\left(a_{k}, b_{k}\right)$ are the linear transformation coefficients.

By minimizing the cost function, the difference between the output image and the input image is minimized to determine the optimal solutions of the linear transformation coefficients as follows:

$$
\begin{gathered}
a_{k}=\left(\Sigma_{k}+\varepsilon E\right)^{-1}\left(\frac{1}{|w|} \Sigma I_{i}^{\text {guide }} \tilde{t}_{i}(x)-\mu_{k} \bar{t}_{k}\right) \\
b_{k}=\bar{t}_{k}-a_{k}^{T} \mu_{k}
\end{gathered}
$$

where $\Sigma_{k}$ is the variance matrix of the guided image $I^{\text {guide }}$ in the window of $w_{k}$, $E$ is a unit matrix, $|w|$ is the number of pixels in the window of $w_{k}, \mu_{k}$ is the mean value of the guided image in the window of $w_{k}, \bar{t}_{k}$ is the mean value of $\tilde{t}(x)$ in the window of $w_{k}, \varepsilon$ is an adjustment parameter to prevent $a_{k}$ from growing too big. 


\subsection{Image defogging}

By using the optimized transmittance $q(x)$, the fog-free image is obtained by the following formula:

$$
J(x)=\frac{I(x)-A}{\max \left(q(x), q_{0}\right)}+A
$$

where the atmospheric light $A$ can be obtained by calculating the position of the brightest $0.1 \%$ pixels in the dark channel image, and then taking the maximum value of the brightness of these pixels in the original image [4]. $q_{0}$ is a lower limit of the transmission to prevent the introduction of noise, usually takes 0.1 .

\section{Edge extraction}

According to the present information, the edge is the basis of image recognition, analysis and understanding. Edge detection is mainly to extract the region where the gray scale changes rapidly, which is shown as high-frequency components in the frequency domain.

The edge extraction is usually done by convolution by using a derivative operator, and the Laplacian operator is one of those most commonly used. It uses zero across points of the second derivative to calculate the edge points, with gradient isotropy, simple calculation and other advantages. However, the Laplacian operator is sensitive to noise, so it is necessary to filter out the noise before edge detection. Generally, the Gaussian filter function and the Laplacian operator are combined together to form the LoG operator as follows [10]:

$$
\nabla G_{\sigma}(x, y)=\frac{1}{\pi \sigma^{4}}\left(\frac{x^{2}+y^{2}}{2 \sigma^{2}}-1\right) \exp \left(\frac{x^{2}+y^{2}}{-2 \sigma^{2}}\right)
$$

where $\sigma$ is the standard deviation of the Gaussian function. With the convolution operation between LoG operator and the image, the zero crossing points are the edge points. The formula is as follows:

$$
\nabla g(x, y)=\nabla G_{\sigma}(x, y) * f(x, y)
$$

\section{Image fusion}

Image fusion is a method to obtain high-quality images by combining useful information and multiple channels, and it can be used to improve image reliability and utilization. In order to highlight the details of the defogged image and improve image clearness, the LOG edge detection features of the original image and the image after the dark channel prior defogging are integrated in a certain proportion, which is shown as:

$$
f(x)=(1-k) J(x)+k g(x)
$$

Where $k(0<k<1)$ is the fusion ratio. The fused image has common characteristics of two images. The size of $k$ determines the effect of fusion. If $k$ is too large, the texture of image after fusion is too detailed to cause the decline of image recognition and understanding. On the contrary, the fusion effect is not obvious and the texture cannot be highlighted if $k$ is too small. 


\section{Experimental results and analysis}

Under the Matlab environment, the original image is convoluted firstly by the LOG operator and the edge is obtained. Then, the initial transmittance is calculated and the atmospheric light is estimated by using dark channel prior knowledge. The radius of the guided filtering window is set and the transmittance is optimized to get the defogged image. Finally, the fusion ratio $k$ is set, and the defogged image and the edge are weighted and summed to obtain the final fused image. Taking a certain aerial image as an example, $w_{k}=20$, $k=0.05$, we can obtain the edge, the defogged image and the fused image as shown in Figure 1.

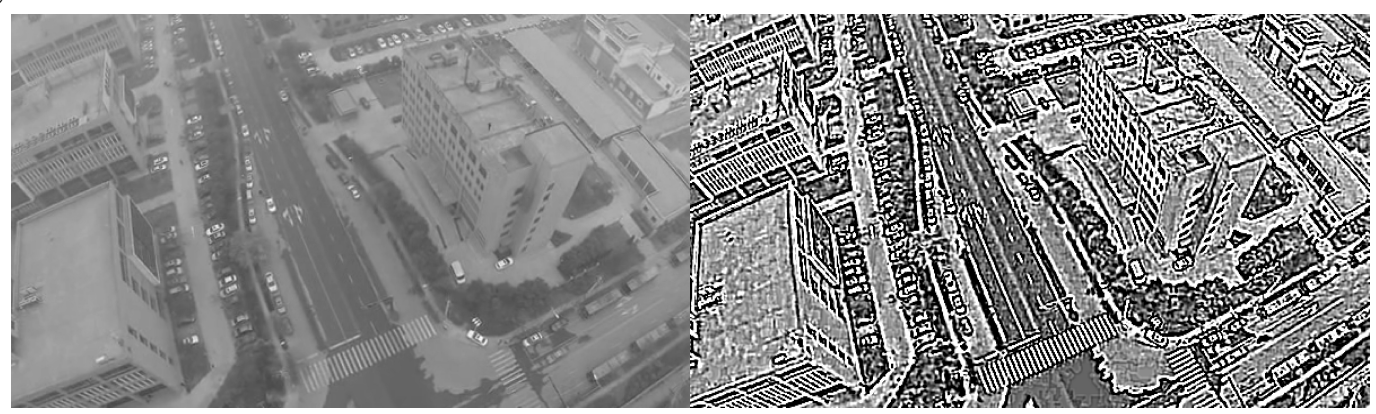

(a) Original Image

(b) Edge of LOG

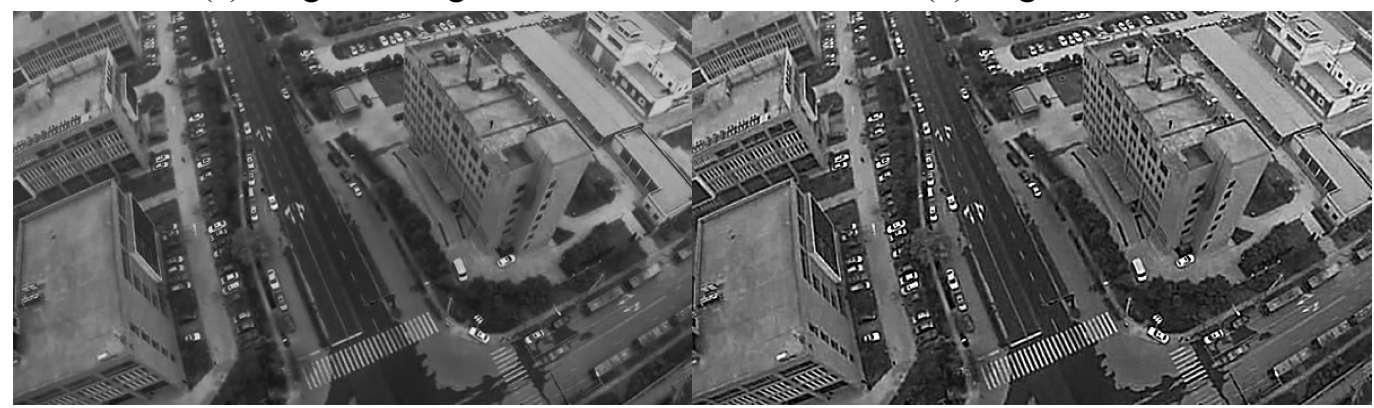

(c) Defogged Image

(d) Fused Image

Figure 1 :Processing Effect of An Aerial Remote Sensing Image

As can be seen from the above figure, compared with the original image, the fused image is intuitively able to reduce the influence of the clouds. Compared with defogged image, the texture details of the original image are highlighted, and the clearness is improved greatly.

In addition to the subjective evaluation by visual observation, there is another method called objective evaluation, which is usually divided into the evaluation of image information amount and the evaluation of image clarity. The indicators of information amount evaluation include gray level entropy and standard deviation. The indicators of clarity evaluation include mean gradient, spatial frequency, Brenner function and TenenGrad function.

In this paper, 20 foggy aerial remote sensing images obtained at different times, locations and from different devices were processed. The range of fusion ratio $k$ was $[0.01,0.20]$. According to calculation, the standard deviation, mean gradient, spatial frequency, Brenner function, and TenenGrad function increased with the increase of $k$. Because the weight of the edge gets greater and image texture becomes more obvious, these parameters representing detail contrast get larger. However, excessive texture will lead to decrease in gray level entropy and the amount of image information. In this paper, the gray level entropy was selected to determine the optimal fusion ratio $k$. The increased percentages of image gray level entropy 
were compared between the fused images and the original images, and those were compared between the fused images and the defogged images as shown in Figure 2.

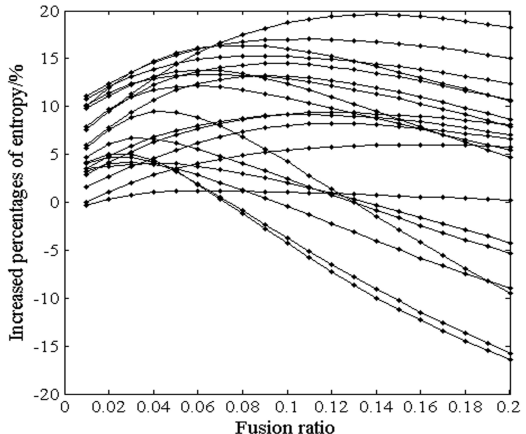

(a) Compared with the Original Image

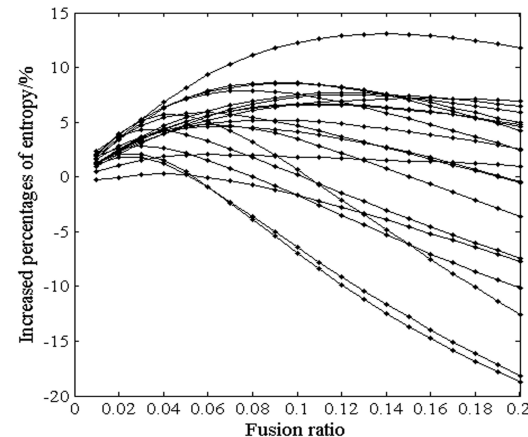

(b) Compared with the Defogged Image

Figure 2: Change Curve of Gray Level Entropy

From the above figures, compared with original images and defogged images, the gray level entropy of most fused images increased, which indicated that the image information amount became larger after fusion. However, with the increase of the fusion ratio, the increased percentage of entropy reached a maximum value and then decreased. When the ratio was too large, the entropy after fusion even reduced. It can be seen from the figures that the maximum values appear when $k$ changes from 0.03 to 0.16 . Because the larger fusion ratio will lead to a decrease of entropy and the image clarity, the best fusion ratio is set to 0.03 . At this time, the information amount of fused images increases compared with the original images, and the clarity will not be affected by excessive texture. Combined with visual experience which is less affected by fog, $k$ is smaller when the maximum value appears. Therefore, for the image with much more fog, $k$ is usually set to be larger than 0.03 , and it's the opposite for the image with less fog.

In order to further illustrate the effectiveness of this method, the evaluation parameters after fusion were selected as the reference standards, which were set to 100 . The average parameters of the 20 original images and the defogged images were calculated when $k=0.03$, as shown in Table 1. The objective evaluation parameters of the defogged images are higher than those of the original images, and the objective evaluation parameters of the fused images are higher than the defogged images. It proves that our method can increase the image information amount and improve the image clarity. The correctness and effectiveness of this method are also verified.

\begin{tabular}{ccccccc}
\hline Indicators & $\begin{array}{c}\text { Gray Level } \\
\text { Entropy }\end{array}$ & $\begin{array}{c}\text { Standard } \\
\text { Deviation }\end{array}$ & Mean Gradient & $\begin{array}{c}\text { Spatial } \\
\text { Frequency }\end{array}$ & Brenner & TenenGrad \\
\hline Original Images & 92.78 & 63.27 & 0.15 & 39.87 & 15.43 & 15.49 \\
Defogged Images & 96.62 & 85.15 & 55.16 & 58.33 & 32.76 & 32.72 \\
Fused Images & 100 & 100 & 100 & 100 & 100 & 100 \\
\hline
\end{tabular}

Table 1: An Objective Evaluation of Fused Images, Original Images and Defogged Images

\section{Conclusion}

In this paper, a new method for the clearance of foggy aerial remote sensing images is proposed. Firstly, the edge features of the original images were obtained, and fog was removed 
by using dark channel prior, and then the edges and results of dark channel prior were fused in a certain proportion to get fused images. Through the subjective and objective evaluation of the images before and after fusion, it shows that the proposed method not only gets a good defogging effect, but also preserves the image texture and improves the image quality. How to obtain the fusion ratio adaptively to improve the image clarity will be the future research directions.

\section{References}

[1] G. H. Zhao, X. L. Zou, C. G. Dai, Y. Fang, H. Chen, H. Wan. An improved method of haze removal for aerial digital image based on dark channel prior [J]. Science of Surveying and Mapping. 38(1), 29-32 (2013) (In Chinese)

[2] A. Galdran, J. Vazquez-Corral, D. Pardo, M. Bertalm'10. Fusion-Based Variational Image Dehazing [J]. IEEE Signal Processing Letters. 24(2), 151-155 (2017)

[3] Y. Lee, K. Hirakawa, T. Nguyen. Joint Defogging and Demosaicking [J]. IEEE Transactions on Image Processing. PP(99), 1-1 (2016)

[4] K. M. He, J. Sun, X. O. Tang. Single image haze removal using dark channel prior [C]. IEEE Conference on Computer Vision and Pattern Recognition. IEEE, pp,1956-1963 (2009)

[5] K. M. He, J. Sun, X. O. Tang. Guided Image Filtering [C]. ECCV'10 Proceedings of the 11th European conference on Computer vision. pp,1-14 (2010)

[6] S. Z. Chen, Z. G. Ren, Q. S. Lian. Single Image Dehazing Algorithm Based on Improved Dark Channel Prior and Guided Filter [J]. Acta Automatica Sinica. 42(3), 455-465 (2016) (In Chinese)

[7] E. J. McCartney. Optics of the Atmosphere: Scattering by Molecules and Particles [M]. John Wiley and Sons Inc, pp,123-129 (1976).

[8] X. Q. Ji. Research on Fast Image Defogging and Visibility Restoration [D]. Changchun: Changchun Institute of Optics, Fine Mechanics and Physics, Chinese Academy of Science, China (2012) (In Chinese)

[9] J. B. Tang, G. B. Zhu, T. Jiang, T. Wang. Research on Single Image Dehazing using Guided Filtering [J]. Science Technology and Engineering. 13(11), 114-119 (2013) (In Chinese)

[10] D. Marr, E. Hlidrith. Theory of Edge Detection [C]. Proceedings of the Royal Society of London, Series B, Biological Sciences. The Royal Society, pp,187-217 (1980) 